suffered severe deterioration of vision before being discovered. Even when patients are originally thought to have escaped ophthalmic complications, unsuspected glaucoma may yet be present with slowly advancing loss of sight. Every patient with facial naevus should have regular ophthalmic supervision.

${ }^{1}$ Schrimer, R, Archiv für Ophthalmologie, 1860, 7, 119.

2 Salus, R, Klinische Monatsblätter für Augenheilkunde, 1923, 71, 305.

${ }^{3}$ Sturge, A, Transactions of the Clinical Society of London, 1879, 12, 162.

${ }^{4}$ Horrocks, P, Transactions of the Ophthalmological Societies of the United Kingdom, 1883, 3, 106.

${ }^{5}$ Weber, J, Proceedings of the Royal Society of Medicine, 1929, 22, 431.

(Accepted 6 October 1978)

Glaucoma Centre, Department of Ophthalmology, King's College Hospital, London SE5 9RS

A O'CONNELL, FRCS, ophthalmic senior registrar

R PITTS CRICK, FRCS, consultant ophthalmic surgeon

\section{Urethral discharge associated with ingested foreign body}

Foreign bodies in the urethra cause a chronic discharge. Usually (excluding renal calculi that have become impacted in the urethra) they have been knowingly inserted per urethra by the patient. An unusual case is discussed in which a foreign body which was accidentally ingested became impacted in the bulbous urethra.

\section{Case report}

A 55-year-old man was admitted with a history of dysuria and pneumaturia for a few weeks. In 1938 he had had an arthrodesis of the right hip for tuberculosis and also an appendicectomy and repair of a left inguinal hernia. He was generally well at the time of presentation. Examination showed no gross abnormality other than mild hypertension $(150 / 100 \mathrm{~mm} \mathrm{Hg})$ and a fixed hip. Culture of the urine produced a heavy growth of Escherichia coli ; chest $x$-ray examination was normal; an intravenous pyelogram showed no gross abnormality of the kidneys, ureter, or bladder; a barium enema showed diverticular disease of the sigmoid colon with a fistula to the bladder. Laparotomy confirmed the diverticulosis and fistula. The sigmoid colon was resected and the fistula closed. No abnormality within the bladder was noted. The patient made a good recovery and was discharged home on the eighth postoperative day with normal micturition, bowel action, and a well-healed wound. The urine was sterile.

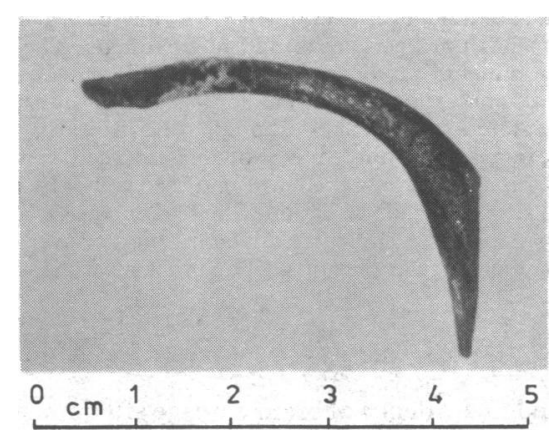

Duck bone removed from urethra.

One month later the patient was well but had developed a moderate urethral discharge. A urine culture was sterile but the discharge grew haemolytic streptococci. Acid-fast bacilli were not seen or cultured. The discharge persisted despite appropriate antibiotic treatment. An intravenous pyelogram, micturating cystogram, and cystoscopy were all normal; a urethrogram showed a diverticulum in the bulbous urethra. At urethroscopy a foreign body was seen in the urethra. When removed it was found to be an animal bone $4-5 \mathrm{~cm}$ long (figure). The discharge immediately ceased and the patient has been well since. In retrospect, he remembered swallowing a duck bone one month before the original pneumaturia.

\section{Comment}

Diverticulosis is the commonest cause of vesicocolic fistula, ${ }^{12}$ but we could find no report of a case in which an ingested foreign body was associated with a fistula. Urethritis is the commonest cause of urethral discharge, and may be associated with an inserted foreign body. ${ }^{3}$ In this patient the duck bone was ingested. It may have caused the vesicocolic fistula or may merely have passed through a fistula caused by diverticulitis. In the early stages of investigation and at operation the bone was not seen, and thus must have passed as far as the bulbous urethra submucously. The case is reported as a most unusual cause of urethral discharge, and possibly of the fistula itself.

1 Slade, N, and Gaches, C, British fournal of Surgery, 1972, 59, 593.

2 Henderson, M A, and Small, W P, British fournal of Urology, 1969, 41, 314.

3 Thomas, W J C, British fournal of Surgery, 1964, 51, 921.

(Accepted 27 September 1978)

Department of Surgery, North Devon Infirmary, Barnstaple, Devon

W N WYKES, MB, senior house officer in surgery

J R BARKER, MB, FRCS, consultant surgeon

\section{Adenosine deaminase activity in pleural effusions: an aid to differential diagnosis}

Adenosine deaminase (ADA) catalyses the conversion of adenosine to inosine. Serum concentrations of this enzyme are high in patients with typhoid fever and infectious mononucleosis. ${ }^{1}$ They are also high in the cerebrospinal fluid of patients with tuberculous meningitis. This helps to differentiate tuberculous from viral lymphocytic meningitis. $^{2}$ We investigated ADA concentrations in tuberculous pleural effusions and in pleural and peritoneal effusions of different aetiology.

\section{Patients, methods, and results}

ADA concentrations were measured in effusions from 96 patients. Of these 96,54 had pleural effusions and 42 had peritoneal effusions. The pleural effusions were due to tuberculous pleurisy (21 cases), metapneumonic pleurisy (15 cases), and primary and metastatic pleuropulmonary malignancies $(18$ cases). The peritoneal effusions were due to cirrhosis and ascites (25 cases) and abdominal primary and metastatic malignancies (17 cases). ADA assay was performed as previously described. ${ }^{2}$ The mean $( \pm S D)$ ADA concentration in tuberculous pleural effusions was $83.04 \mathrm{IU} / 1 \pm 25.51$ (figure). This was much higher $(P<0.01$ by Student's $t$ test) than in metapneumonic pleurisy $(17.26 \mathrm{IU} / 1 \pm 7 \cdot 51)$ and pleuropulmonary malignancies $(15.54 \mathrm{IU} / 1$ $\pm 6.56)$. The mean concentrations in peritoneal effusions due to cirrhosis and ascites were $4.06 \mathrm{IU} / 1 \pm 3.47$ and in effusions due to malignancies $11.46 \mathrm{IU} / 1$ $\pm 6 \cdot 74$-both very low. The variance analysis in the five groups showed a highly significant difference $(P<0.001)$.

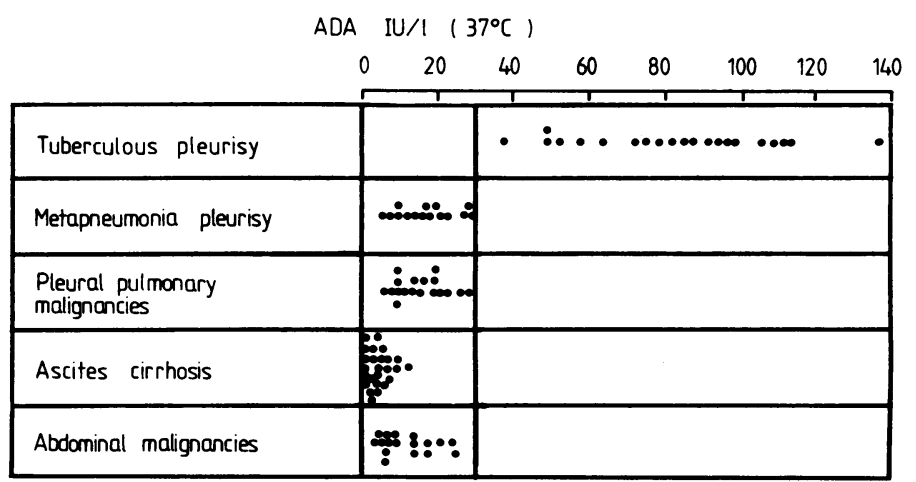

ADA concentrations in pleural and peritoneal effusions. 


\section{Comment}

ADA deficiency has been reported in patients with combined immunodeficiency (CID), an association which is inherited as an autosomal recessive trait. ${ }^{3}$ To try to explain the exact mechanism by which ADA deficiency causes lymphocyte impairment it was suggested that ADA had a crucial function in the differentiation or proliferation, or both, of lymphoid cells. Indeed, lymphocytes from patients with CID-ADA deficiency fail to be transformed in plant mitogen and allogeneic cell-stimulated cultures. ${ }^{4}$ This inhibition has been attributed to high concentrations of adenosine or to a secondary pyrimidine starvation. Although normal lymphocytes are inhibited when treated with adenosine deaminase inhibitors, however, much higher adenosine concentrations are required than those found in patients' plasma. On the other hand, the addition of uridine does not restore the in-vitro response to mitogens of ADA-CID lymphocytes. A possible inhibitory role has been ascribed to an increased cyclic AMP synthesis. ${ }^{4}$ More recently a toxic effect of deoxyadenosine has been suggested. ${ }^{5}$ We can understand how ADA concentrations increase in typhoid fever and tuberculosis, in which host defences are mostly a function of cellmediated immunity. In infectious mononucleosis, after the entry of Epstein-Barr virus into B cells, a strong, prolonged $\mathrm{T}$-cell response occurs.

The augmented activity of adenosine deaminase in vivo owing to antigenic stimuli underlines the importance of this enzyme to rapid proliferating cells and agrees well with the negative response observed in CID-ADA deficiency. The increased enzymatic activity in tuberculous pleurisy further supports the view of a causal relationship between adenosine deaminase and $T$-cell response. Cellular activation could be responsible for an augmented enzymatic activity to avoid the accumulation of toxic metabolites, but an increased demand for energy might be the explanation. But why, in human pathology, does B cell activation seem not to be responsible for the same phenomenon? Is ADA a new marker for delayed immunity only?

ADA assay seems to be a simple clinical test in the differential diagnosis of exudative pleurisy, particularly when the results of other laboratory and clinical tests are negative.

1 Giusti, G, in Methods of Enzymatic Analysis, ed H U Bergmeyer. New York, Academic Press Inc, 1974.

2 Piras, M A, and Gakis, C, Enzyme, 1973, 14, 311

${ }^{3}$ Scott, C R, Chen, S-H, and Giblett, E R, fournal of Clinical Investigation, $1974,53,1194$

4 Schmalstieg, F C, et al, fournal of Pediatrics, 1977, 91, 48.

5 Simmonds, H A, Panayi, G S, and Corrigall, V, Lancet, 1978, 1, 60.

(Accepted 18 October 1978)

Clinic of Infectious Diseases, University of Sassari, Italy

M A PIRAS, MD, assistant

C GAKIS, MD, assistant

M BUDRONI, MD, assistan

G ANDREONI, professor of infectious diseases

\section{CAT brain scans after central nervous system prophylaxis for acute lymphoblastic leukaemia}

Prophylactic treatment of the central nervous system in children with acute lymphoblastic leukaemia (ALL) has been the biggest single factor in increasing survival in the last 15 years. Although the neurotoxic complications of both radiation and intrathecal chemotherapy have usually been described in patients who have had large doses or prolonged exposure, or both, concern has recently been expressed about the sequelae of standard CNS prophylaxis, after reports of abnormalities on cranial computerised tomographic scans (CAT)..$^{1-3}$ We therefore performed CAT scans on children who had received CNS prophylaxis at the beginning of their illness and who had not had meningeal involvement.

\section{Patients, methods, and results}

CAT was performed on 27 children aged 20 months to 7 years (mean 4.4 years) after CNS prophylaxis for ALL. The children were aged between 16 months and 11 years (mean 5.8 years) at the time of treatment. In all but two cases radiation was given from a Philips $250 \mathrm{KV}$ (that is, orthovoltage) source with a mean dose of 2054 rads in 10-15 fractions over 11-22 days (supposedly biologically equivalent to 2500 rads from a megavoltage source). The mean dose of intrathecal methotrexate (ITMTX) was $62.5 \mathrm{mg} / \mathrm{m}^{2}$. The largest dose given over the longest period was $10 \mathrm{mg} / \mathrm{m}^{2} \times 11$ doses between weeks $17-55$ of treatment. All children were well at the time of CAT.

Ventricular size was measured in two ways. Firstly, the diameter of the frontal horns at two sites was compared with that of the brain at the same point to give (1) the bifrontal ratio, and (2) the bicaudate ratio. ${ }^{4}$ Secondly, by the method described by Enzmann and Lane. ${ }^{5}$

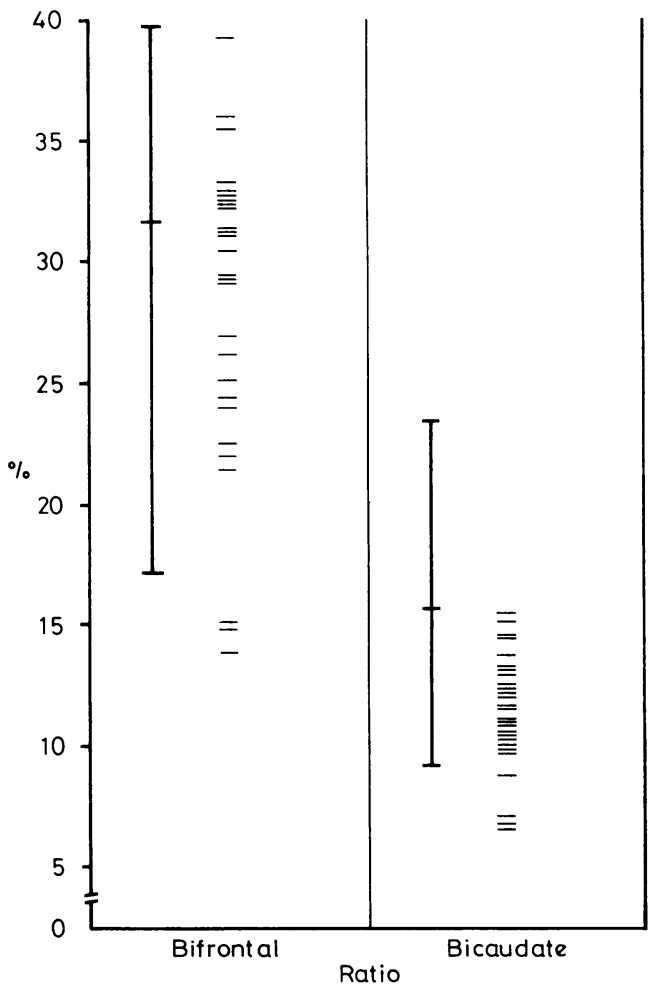

Ventricle/brain ratios in 27 children compared with the mean and range in normal adults.

None of the scans showed abnormal low density areas or calcification. In four patients the sulci or subarachnoid spaces were more obvious than in the others, but in none were they considered definitely outside the normal range. One boy, who was born prematurely and known to be of low intelligence before treatment, had dilated ventricles. His performance has not deteriorated and the dilatation has not been atrributed to treatment. The results of the ventricular measurements of the remaining 27 children are shown in the figure with a mean and range for normal adults. The bicaudate diameter of one patient is separated from the rest, though still within the upper limit of normal. This scan was the only one showing enlarged ventricles by the criteria of Enzmann and Lane. ${ }^{5}$ The child had received only $22 \mathrm{mg} / \mathrm{m}^{2}$ total dose of ITMTX and 2000 rads radiation. She had extensive retinal haemorrhage at diagnosis, and the ventricular dilatation might have been the result of intracranial haemorrhage at that time. The other 26 scans were normal.

\section{Comment}

We have found no evidence of brain damage after our standard form of CNS prophylaxis as shown by the relatively crude instrument of CAT. This result is at variance with that reported by Peylan-Ramu et al, ${ }^{1}$ who studied asymptomatic patients similar to ours and found that $53 \%$ had abnormal CAT scans. The biggest single difference between the two series was that they gave monthly intrathecal chemotherapy throughout maintenance. An alternative but less likely reason was their use of megavoltage as opposed to orthovoltage radiation. Enzmann and Lane ${ }^{2}$ reported abnormal CAT scans in two of nine patients who had leukaemia but no evidence of CNS disease. Ochs et $a l^{3}$ evaluated 26 asymptomatic children who had received CNS prophylaxis with MTX alone: only one patient had an abnormal CAT scan and she was thought to have had CNS leukaemia. Further prospective studies are needed to identify those elements which may be responsible for permanent damage. We have found no evidence, 